\title{
Overexpression of satellite alpha transcripts leads to chromosomal instability via segregation errors at specific chromosomes
}

\author{
KOSUKE ICHIDA $^{1}$, KOICHI SUZUKI ${ }^{1}$, TARO FUKUI ${ }^{1}$, YUJI TAKAYAMA ${ }^{1}$, NAO KAKIZAWA ${ }^{1}$, \\ FUMIAKI WATANABE ${ }^{1}$, HIDEKI ISHIKAWA ${ }^{1}$, YUTA MUTO ${ }^{1}$, TAKAHARU KATO ${ }^{1}$, MASAAKI SAITO ${ }^{1}$, \\ KAZUSHIGE FUTSUHARA ${ }^{1}$, YASUYUKI MIYAKURA ${ }^{1}$, HIROSHI NODA ${ }^{1}$, \\ TSUKASA OHMORI $^{2}$, FUMIO KONISHI ${ }^{3}$ and TOSHIKI RIKIYAMA ${ }^{1}$ \\ ${ }^{1}$ Department of Surgery, Saitama Medical Center, Jichi Medical University, Saitama-shi, Saitama 330-8503; \\ ${ }^{2}$ Department of Biochemistry, Jichi Medical University, Shimotsuke-shi, Tochigi 329-0498; \\ ${ }^{3}$ Nerima-Hikarigaoka Hospital, Tokyo 179-0072, Japan
}

Received December 11, 2017; Accepted February 22, 2018

DOI: 10.3892/ijo.2018.4321

\begin{abstract}
The impairment of the stability of the chromosomal structure facilitates the abnormal segregation of chromosomes, thus increasing the risk of carcinogenesis. Chromosomal stability during segregation is managed by appropriate methylation at the centromere of chromosomes. Insufficient methylation, or hypomethylation, results in chromosomal instability. The centromere consists of satellite alpha repetitive sequences, which are ideal targets for DNA hypomethylation, resulting in the overexpression of satellite alpha transcript (SAT). The overexpression of SAT has been reported to induce the abnormal segregation of chromosomes. In this study, we verified the oncogenic pathway via chromosomal instability involving DNA hypomethylation and the overexpression of SAT. For this purpose, we constructed lentiviral vectors expressing SAT and control viruses and then infected human mammary epithelial cells with these vectors. The copy number alterations and segregation errors of chromosomes were evaluated by microarray-based comparative genomic hybridization (array CGH) and immunocytochemistry, respectively. The levels of hypomethylation of satellite alpha sequences were determined by MethyLight polymerase chain reaction. Clinical specimens from 45 patients with breast cancer were recruited to verify the data in vitro. The results of immunocytochemistry revealed that the incidence of segregation errors was significantly higher in the cells overexpressing SAT than in the controls. An array CGH identified the specific chromosomes of $8 \mathrm{q}$ and $20 \mathrm{q}$ as frequent sites of copy number alterations in cells with SAT overexpression, although no such
\end{abstract}

Correspondence to: Dr Koichi Suzuki, Department of Surgery, Saitama Medical Center, Jichi Medical University, 1-847 Amanuma-cho, Omiya-ku, Saitama-shi, Saitama 330-8503, Japan

E-mail: ksuzbnhm@yahoo.co.jp

Key words: satellite alpha transcript, chromosomal instability, DNA hypomethylation, centromere, development of breast cancer sites were noted in the controls, which was consistent with the data from clinical specimens. A regression analysis revealed that the expression of SAT was significantly associated with the levels of hypomethylation of satellite alpha sequences. On the whole, the overexpression of SAT led to chromosomal instability via segregation errors at specific chromosomes in connection with DNA hypomethylation, which was also recognized in clinical specimens of patients with breast cancer. Thus, this oncogenic pathway may be involved in the development of breast cancer.

\section{Introduction}

Epigenetic alterations have been identified in many types of human cancer in connection with carcinogenesis without genetic sequence abnormalities (1-4). DNA methylation has long been investigated as the process of carcinogenesis induced by epigenetic alterations. While regional methylation alterations have been recognized to induce the transcription silencing of tumor suppressor genes at the $\mathrm{CpG}$ island of the promoter region (5-7), global hypomethylation alterations have not received much attention, with no studies to date investigating the role of such changes in the process of cancer development and progression, at least to the best of our knowledge.

DNA repetitive sequences are globally distributed in the human genome and are ideal targets for hypomethylation. These sequences are involved in tumor progression in ovarian epithelial carcinomas and hepatocellular carcinomas and are considered to be a better predictor of a poor survival than conventional markers $(8,9)$. LINE-1 is a transposable element in the human genome comprising a repetitive sequence. The hypomethylation of LINE-1 has been reported to be significantly associated with chromosomal instability in gastrointestinal stromal tumors (10). Experimental studies using mouse models have provided evidence that global hypomethylation and chromosomal instability are associated with cancer development $(11,12)$. We have previously demonstrated that DNA hypomethylation is enhanced by chronic gastritis mediated by Helicobacter pylori infection. In addition, aging 
is also a contributory factor to enhanced DNA hypomethylation in colon and stomach cancers $(13,14)$. These data suggest that global DNA hypomethylation plays a crucial role in carcinogenesis by affecting the machinery that maintain chromosomal stability. However, the mechanisms through which global DNA hypomethylation induces chromosomal instability remain unclear.

Satellite DNA consists of highly repetitive non-coding sequences. While LINE-1 is globally distributed in the human genome, satellite DNA is mainly located in the centromeric and pericentromeric regions of the chromosomes. Although satellite DNA can be observed in almost all eukaryotic genomic DNA, the sequences are species-specific (15). In the human genome, the centromeric region consists of 171-base monomeric arrays, termed satellite alphas. For many years, this non-coding region was not considered to play any particular role, as it was hardly transcribed due to the heterochromatin structure of its location; by contrast, the appropriate transcription of satellite regions has been reported to be essential for accurate chromosomal segregation (16-19). The over-transcription from satellite DNA can be observed in various epithelial tumors (20), and the overexpression of satellite RNA has been found to induce the abnormal segregation of chromosomes in experimental studies $(21,22)$.

However, the inhibition of satellite alpha transcription has also been reported to lead to the disruption of kinetochore formation, which is a prerequisite for chromosomal segregation (19). Satellite DNA and its transcripts are, therefore, important elements for regulating cell division, and the disruption of this machinery, known as aberrant transcription, can disrupt chromosomal stability. Furthermore, aberrant transcription from the satellite regions in cancer cells has been shown to be induced by epigenetic alterations of global DNA hypomethylation (23). Several studies have demonstrated that demethylation agents induce genome-wide DNA hypomethylation, including in the satellite regions, thereby resulting in the overexpression of satellite RNA (24-26).

In the present study, we addressed satellite DNA as repetitive sequences, which are ideal targets for DNA hypomethylation. We also verified the oncogenic pathway in which aberrant transcription from the satellite regions induced by DNA hypomethylation leads to chromosomal instability, consequently resulting in carcinogenesis.

\section{Materials and methods}

Patients and specimens. Samples of tumor tissue (TT) analyzed in this study were obtained from 45 patients who underwent mastectomy for breast cancer from July, 2015 to July, 2016 at Saitama Medical Center, Jichi Medical University, Saitama-shi, Japan. Non-cancerous mammary gland tissues (NMTs) corresponding TT were also obtained from 35 of the 45 patients with breast cancer. The clinical and pathological findings are presented in Table I. NMTs were defined as tissues $\geq 2 \mathrm{~cm}$ macroscopically distant from the tumor margin and microscopically identified as normal mammary gland by the histological examination of hematoxylin and eosin (H\&E)-stained sections. Corresponding NMTs were obtained from the resected specimen prior to the sampling of TT to avoid contamination by tumor cells. Tissue specimens
Table I. Clinicopathological features of breast cancer specimens.

\begin{tabular}{|c|c|}
\hline Characteristic & $\mathrm{n}=45$ \\
\hline Mean age $( \pm S D)$ & $59.5 \pm 13.1$ \\
\hline Mean BMI $( \pm$ SD $)$ & $23.5 \pm 4.0$ \\
\hline $\mathrm{T}(\mathrm{Tis} / \mathrm{T} 1-3 / \mathrm{T} 4)$ & $3 / 39 / 3$ \\
\hline $\mathrm{N}$ (negative/positive) & $29 / 16$ \\
\hline M (negative/positive) & $41 / 3$ \\
\hline $\mathrm{ER}^{\mathrm{a}}$ (negative/positive) & $14 / 28$ \\
\hline HER2 a (negative/positive) & $27 / 15$ \\
\hline Bilateral breast tumors (negative/positive) & $39 / 6$ \\
\hline Malignancies in other organs (negative/positive) & $39 / 6$ \\
\hline
\end{tabular}

BMI, body mass index; Tis, tumor in situ; ER, estrogen receptor; HER2, human epidermal growth factor receptor type2. ${ }^{\mathrm{a}} \mathrm{Cases}$ of Tis $(n=3)$ were excluded.

were immediately soaked in RNAlater (Ambion, Austin, TX, USA) and stored at $-80^{\circ} \mathrm{C}$ after the RNAlater solution was removed. This study was approved by the Research Ethics Committee at Jichi Medical University. Written informed consent was obtained from each study participant.

Cell culture and viral transfection. Human mammary epithelial cells (HMEpCs) were obtained from Cell Applications, Inc. (San Diego, CA, USA) and maintained in MammaryLife Medium Complete kit (Lifeline Cell Technology, Frederick, $\mathrm{MD}, \mathrm{USA}$ ). The cells were cultured at $37^{\circ} \mathrm{C}$ in a $5 \% \mathrm{CO}_{2}$ atmosphere.

The plasmid vector, p156RRL-EF1a-GFP-U3H1SatA, which was previously described and used (21), expressing satellite alpha transcript (SAT) and green fluorescent protein (GFP) mRNA was a gift from Inder Verma (Addgene plasmid \#41795). To prepare a control vector, the plasmid was treated with restriction enzymes so that SAT was not expressed. The diagrams of the two vectors are presented in Fig. 1.

Lentiviral particles were produced using the two plasmid vectors with packaging plasmids as pLP1, pLP2 (Invitrogen Corp., Grand Island, NY, USA) and pLP/VSVG (Clontech, Mountain View, CA, USA). These packaging plasmids supply structural and replication proteins required to produce a functional virus. The lentiviral vectors titers carrying GFP were measured by infection of $4 \times 10^{5}$ HMEpCs. GFP expression was measured by a fluorescence-activated cell sorting (FACS) analysis (BD Biosciences, Mountain View, CA, USA) at $48 \mathrm{~h}$ after infection. Typically, $10 \mu \mathrm{l}$ of concentrated vector solution was able to transduce GFP in $\sim 10 \%$ of the cells, and the vector titers for HMEpCs were considered to be $4 \times 10^{6}$ cells $/ \mathrm{ml}$. Viral infections of HMEpCs were performed several times, and the passage number of the cells for transfections was unified. The infected cells were sorted by detecting GFP using FACS in order to increase the proportion of truly transfected cells. With this protocol, we deemed that we had mostly used transfected cells in downstream experiments. We prepared two types of HMEpC as controls: Cells infected with satellite alpha-negative vectors named Control vector and uninfected 

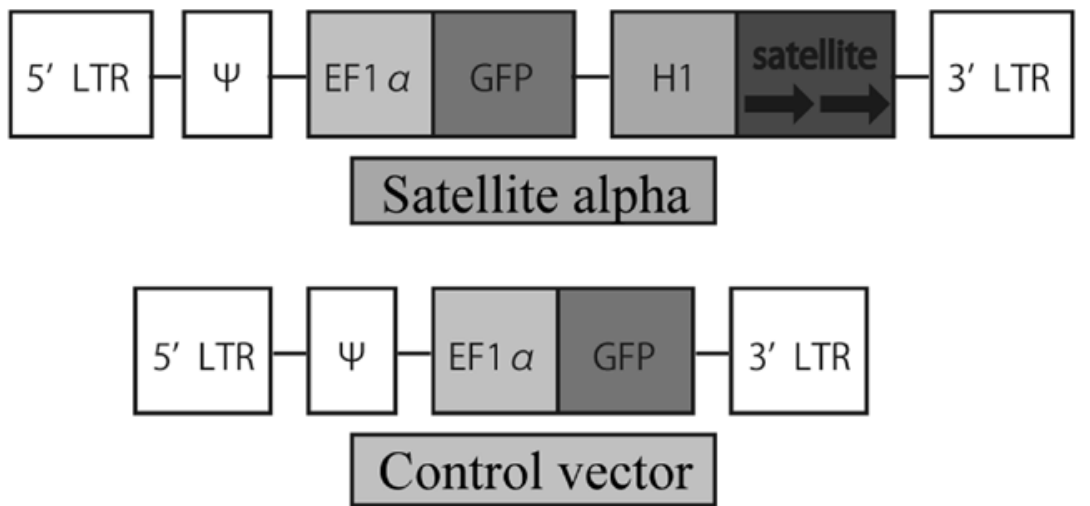

Figure 1. Diagrams of the lentiviral vector expressing SAT (arrows) and the control vector.

Table II. PCR primers for RNA transcript expression.

\begin{tabular}{lc}
\hline Target & \multicolumn{1}{c}{ Sequences (5'-3') } \\
\hline Satellite alpha & F: AAGGTCAATGGCAGAAAAGAA \\
& R: CAACGAAGGCCACAAGATGTC \\
Beta-actin & F: CCATCATGAAGTGTGACGTGG \\
(as an internal & R: GTCCGCCTAGAAGCATTTGCG \\
control) &
\end{tabular}

PCR, polymerase chain reaction; F, forward; $\mathrm{R}$, reverse.

cells named negative control (NC). Both cells were treated in a similar manner to the cells infected with satellite alpha-positive vectors named Satellite alpha, apart from the transfection vectors used.

RNA extraction. Total RNA was extracted from the samples using the Illustra RNAspin Mini RNA Isolation kit (GE Healthcare UK, Buckinghamshire, UK) according to the manufacturer's instructions. To assess the RNA quality and yield, A260/A280 and A260/A230 ratios for RNA preparation samples were analyzed with a NanoDrop ND-1000 spectrophotometer (NanoDrop Technologies, Inc., Wilmington, DE, USA). RNA with an A260/A280 ratio of $\geq 1.8$ was used for subsequent experiments.

Reverse transcription-quantitative polymerase chain reaction (RT-qPCR) assay for RNA transcript expression. Reverse transcription (RT) was performed using the High Capacity RNA-to-cDNA kit (Applied Biosystems, Carlsbad, CA, USA). RT-qPCR assays were performed using SYBR-Green technology and SYBR Premix Ex Taq (Tli RNaseH Plus) on the QuantStudio 12K Flex Real-Time PCR system (Applied Biosystems). The thermal cycling conditions were as follows: $95^{\circ} \mathrm{C}$ for $10 \mathrm{~min}$, followed by 40 cycles of $95^{\circ} \mathrm{C}$ for $15 \mathrm{sec}$ and $56^{\circ} \mathrm{C}$ for $1 \mathrm{~min}$. The expression of each gene was determined using the fluorescence intensity measurements from the QuantStudio 12K Flex Data Analysis software program. A beta-actin (ACTB) fragment was amplified as an internal control. The primer sequences are summarized in Table II. RT-qPCR assays were repeated twice per sample.
DNA extraction and bisulfite modification. The dissected tissues or cultured cells were placed in buffered proteinase $\mathrm{K}$ solution at $56^{\circ} \mathrm{C}$ for $3 \mathrm{~h}$. Genomic DNA was isolated and purified using an EZ1 Advanced XL and an EZ1 DNA Tissue kit (Qiagen, Hilden, Germany) according to the manufacturer's instructions. DNA purity was assessed by the NanoDrop ND-1000 spectrophotometer (NanoDrop Technologies, Inc.) at absorbances of 260 and $280 \mathrm{~nm}$. The A260/A280 ratio exceeded 1.8 in all instances. The sodium bisulfite conversion of genomic DNA was performed using an EpiTect Bisulfite kit (Qiagen). DNA quantities of $400 \mathrm{ng}$ in a volume of up to $40 \mu \mathrm{l}$ were processed using this standard protocol. The treatment of genomic DNA with sodium bisulfite converted unmethylated (not methylated) cytosine to uracil, which was then converted to thymidine during subsequent PCR steps. This process revealed the sequence differences between the methylated and unmethylated DNA.

MethyLight methods. Following bisulfite modification, each sample was examined using MethyLight technology for satellite alpha sequences. Two sets of primers and probes specifically designed to bind to bisulfite-converted DNA were used in the reaction: Satellite alpha primers and a probe for unmethylated target analyses (unmethylated reaction), and Alu-C primers and a probe for the reference locus (normalization control reaction), as previously described (27). The primer and probe sequences are summarized in Table III. Whole-genome amplification provided fully unmethylated DNA obtained from HMEpC DNA, which served as the demethylation constant reference that enabled the determination of the relative demethylation level (RDL). We defined the $\mathrm{RDL}$ as (satellite alpha reaction/Alu-C reaction) sample/(satellite alpha reaction/Alu-C reaction) fully unmethylated control DNA. In each MethyLight reaction, $1 \mu \mathrm{l}$ of bisulfite-modified DNA solution was used. Thermal cycling was initiated with a denaturation step at $95^{\circ} \mathrm{C}$ for $10 \mathrm{sec}$, followed by 50 cycles of $95^{\circ} \mathrm{C}$ for $5 \mathrm{sec}$ and $60^{\circ} \mathrm{C}$ for $30 \mathrm{sec}$. PCR was performed on a QuantStudio 12K Flex Real-Time PCR System with a final reaction volume of $25 \mu$ l containing Premix Ex Taq (Takara Bio, Inc., Otsu, Japan), $600 \mathrm{nM}$ of each primer and $200 \mathrm{nM}$ of the probe.

Microarray-based comparative genomic hybridization (array CGH). Array $\mathrm{CGH}$ was performed using a SurePrint G3 
Table III. PCR primers and TaqMan probes for the MethyLight method.

\begin{tabular}{lll}
\hline Target & & \multicolumn{1}{c}{ Sequences $\left(5^{\prime}-3{ }^{\prime}\right)$} \\
\hline Alu-C & Forward: & GGTTAGGTATAGTGGTTTATATTTGTAATTTTAGTA \\
& Reverse: & ATTAACTAAACTAATCTTAAACTCCTAACCTCA \\
& Probe: & FAM-CCTACCTTAACCTCCC-MGB \\
Satellite & Forward: & TTGATGGAGTATTTTTAAAATATATGTTTTGTAGT \\
alpha & Reverse: & AAATTCTAAAAATATTCCTCTTCAATTACATAAA \\
& Probe: & FAM-TTTATCCCATTTCCAACAAA-MGB \\
\hline
\end{tabular}

PCR, polymerase chain reaction.

Human CGH Microarray kit, 8x60K (Agilent Technologies Inc., Palo Alto, CA, USA). Labeling and hybridization were performed using a SureTag DNA Labeling kit and Oligo aCGH/ChiP-on-chip Hybridization kit (both from Agilent Technologies Inc.) according to the protocol provided by Agilent Technologies Inc. (Protocol v7.3, Oct. 2015). In brief, $\geq 0.2 \mu \mathrm{g}$ of DNA extracted from the tumor samples and an equal amount of control DNA were digested with AluI and $R s a \mathrm{I}$ for $2 \mathrm{~h}$ at $37^{\circ} \mathrm{C}$. The digested DNA was labeled by random priming. The tumor DNA and control DNA were labeled with Cy5-dUTP and Cy3-dUTP, respectively. The labeled products were purified using an Amicon Ultra-0.5 Centrifugal Filter Unit with an Ultracel-30 membrane (Millipore, Billerica, MA, USA) and concentrated to $9.5 \mu 1$. Dye incorporation and the DNA yield were checked with a NanoDrop spectrophotometer. Equal amounts of genomic DNA extracted from tumor samples and control DNAs were mixed with human Cot-1 DNA, dissolved in hybridization buffer (Agilent Technologies Inc.), denatured, and hybridized to the $\mathrm{CGH}$ array at $67^{\circ} \mathrm{C}$ for $24 \mathrm{~h}$. Following hybridization, the microarrays were washed with Oligo aCGH/ChIP-on-Chip Wash Buffer (Agilent Technologies Inc.). After washing, the slides were scanned on an Agilent Technologies Microarray scanner. Microarray images were analyzed using the Feature Extraction software program. v.12.0.3.1 (Agilent Technologies Inc.), and the resulting data were subsequently imported into the Agilent Cytogenomics software program, v.3.0.1.1.

Immunocytochemistry. The cells were cultured on cover glasses in well plates. The prepared cover glasses were fixed in $4 \%$ paraformaldehyde/PBS at room temperature for $15 \mathrm{~min}$ and then washed 3 times with PBS. The cells were permeabilized with $0.2 \%$ Triton X-100 (Agilent Technologies Inc.) at room temperature for $5 \mathrm{~min}$ and blocked with $10 \%$ Normal Goat Serum (Thermo Fisher Scientific, Inc., Waltham, MA, USA) at room temperature for $30 \mathrm{~min}$. The cells were washed 3 times with PBS and incubated with anti- $\alpha$-tubulin (\#ab52866; Abcam, Cambridge, UK; 1:400 dilution) at room temperature $90 \mathrm{~min}$. Following extensive washing with PBS, the samples were incubated with Alexa-594-conjugated antirabbit IgG secondary antibody (\#ab150080; Abcam; 1:500 dilution) and Hoechst 33342 (Thermo Fisher Scientific, Inc.; $10 \mu \mathrm{M}$ ) for nuclear staining at room temperature for $60 \mathrm{~min}$. The cover glasses were washed 3 times with PBS and mounted in ProLong Diamond Antifade Mountant (Thermo Fisher
Scientific, Inc.) and sealed with nail polish. Images were acquired using a Keyence BZ-X700 fluorescence microscope (Keyence, Osaka, Japan).

Statistical analyses. All statistical analyses were performed using the StatView version 5.0 software program (SAS Institute, Cary, NC, USA). When necessary, the differences in qualitative variables were evaluated using either the $\chi^{2}$ test or Fisher's exact test. Continuous variables were compared using an analysis of variance (ANOVA) with Tukey-Kramer test and Student's t-test. The correlation between the two variables was evaluated by Pearson's correlation coefficient. All reported P-values were two-sided, and P-values $<0.05$ were considered to represent a statistically significant result.

\section{Results}

RDL of satellite alpha in NMT and TT. We determined the correlation between the DNA hypomethylation levels of satellite region and the expression of SAT in breast cancer and the NMTs corresponding TT. We evaluated the RDL of satellite alpha in the NMTs and TT from 45 patients with breast cancer using the MethyLight method and measured the expression of SAT by RT-qPCR. Pearson's correlation coefficient revealed that the SAT expression significantly correlated with the levels of hypomethylation of satellite alpha sequences, not only in TT, but also in NMTs (Fig. 2). These data indicated that the expression of SAT was enhanced at the centromeric region where the methylation of satellite alpha was low, even in NMTs surrounding TT, suggesting that changes in hypomethylation are involved in breast cancer development in conjunction with the aberrant expression of SAT.

Array CGH of clinical specimens with a high and low SAT expression. To determine whether or not the overexpression of SAT induces chromosomal instability in breast cancer, an array CGH analysis was carried out to detect copy number alterations in 8 patients, 4 of whom exhibited a high expression of SAT and the other 4 of whom had a low expression of SAT (Fig. 3A). Table IV presents the clinicopathological characteristics of the 8 patients. Copy number alterations were compared between these patients. Array CGH was used to compare the copy number alterations between these patients (Fig. 3B). The results revealed a difference in the number and location of the copy number alterations, but no significant differences were noted in 


\section{Tumor tissue}

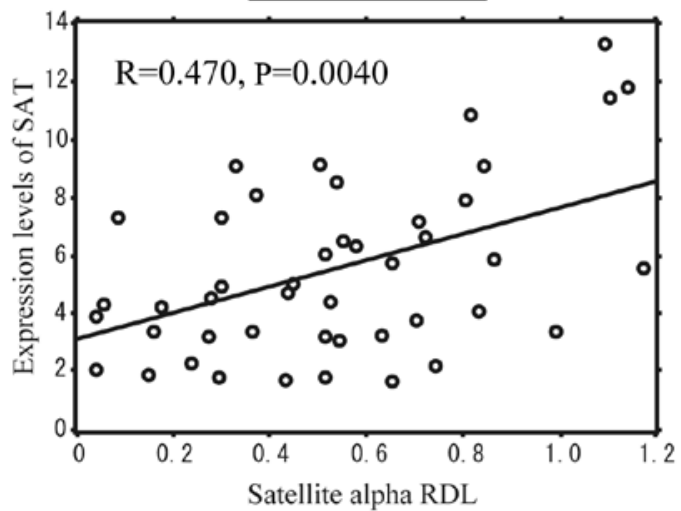

Normal tissue

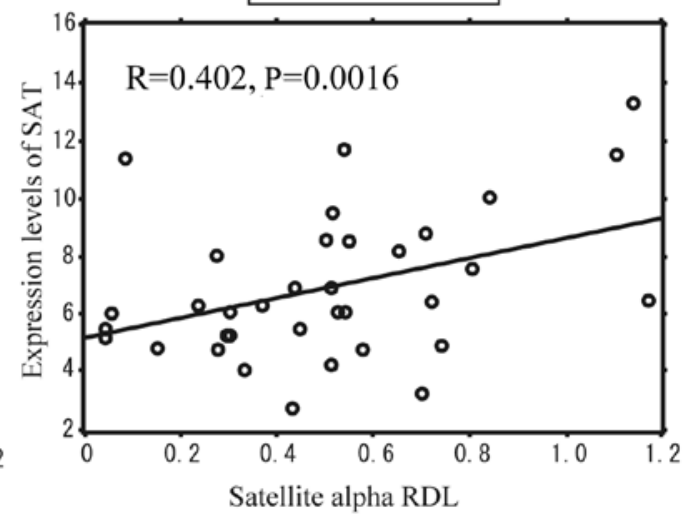

Figure 2. Correlation between the hypomethylation of satellite alpha and the expression of SAT. RDL, relative demethylation level. Circle plots represent satellite alpha RDL and the expression of SAT. Pearson's correlation coefficient (R) and P-values are shown in the upper part of each image. The RDL is defined as (satellite alpha reaction/Alu-C reaction) sample/(satellite alpha reaction/Alu-C reaction) fully unmethylated control DNA.

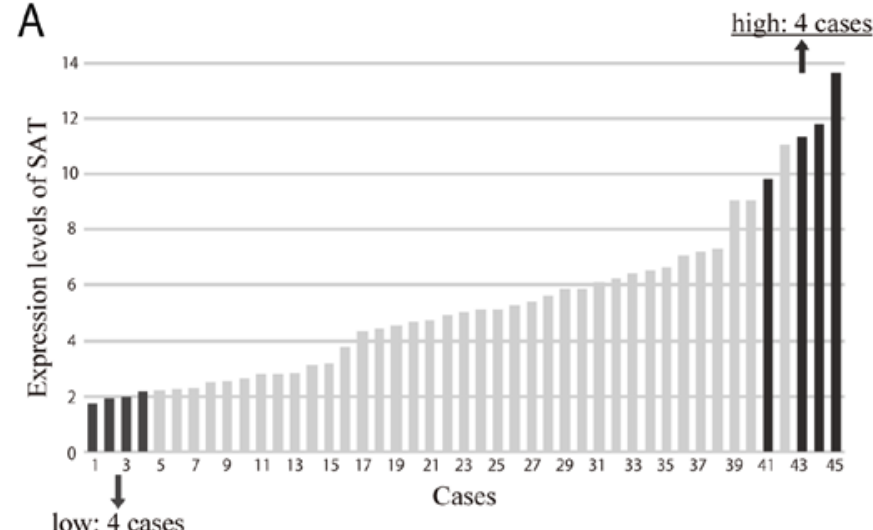

low: 4 cases

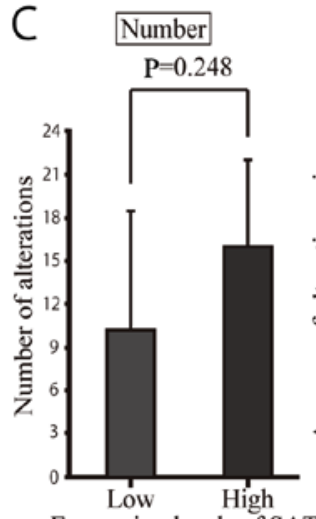

Expression levels of SAT

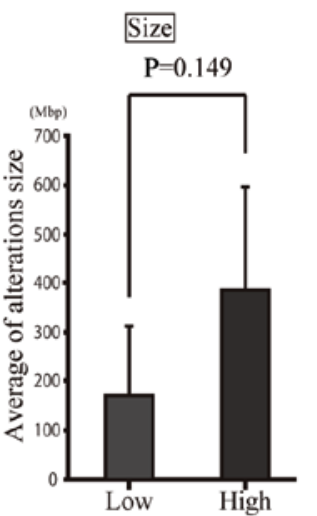

Expression levels of SAT

\section{B SAT low: 4 cases}

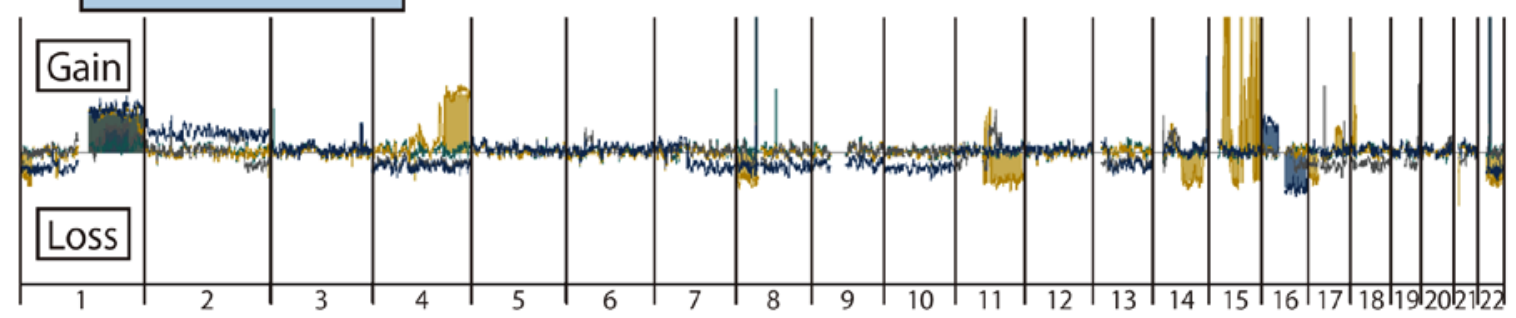

\section{SAT high: 4 cases}

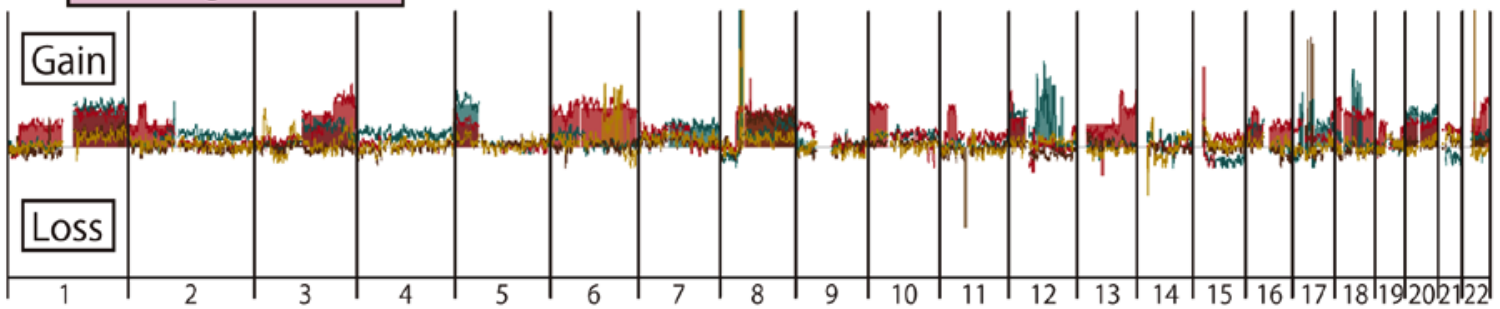

Figure 3. The expression of satellite alpha transcript (SAT) and chromosomal instability in clinical specimens. (A) Distribution of the expression of SAT in 45 tumor specimens in increasing order of expression. (B) Copy number alteration patterns of autosomal chromosomes detected by array CGH. Gains are shown in the upper area and losses in the lower area. Patients are distinguished by different colors. Vertical lines indicate the boundaries of chromosomes. Numbers represent the chromosome number. In each chromosome area, the short arm is located on the left side, and the long arm is on the right side. (C) Comparisons of the number and region size of alterations between high- and low-SAT expression groups. Error bars represent the standard deviation.

the number or size of the regions with copy number alterations based on SAT expression (Fig. 3C).
Mitotic errors in satellite alpha-transfected cells. To examine whether or not the overexpression of SAT induces chromosomal 

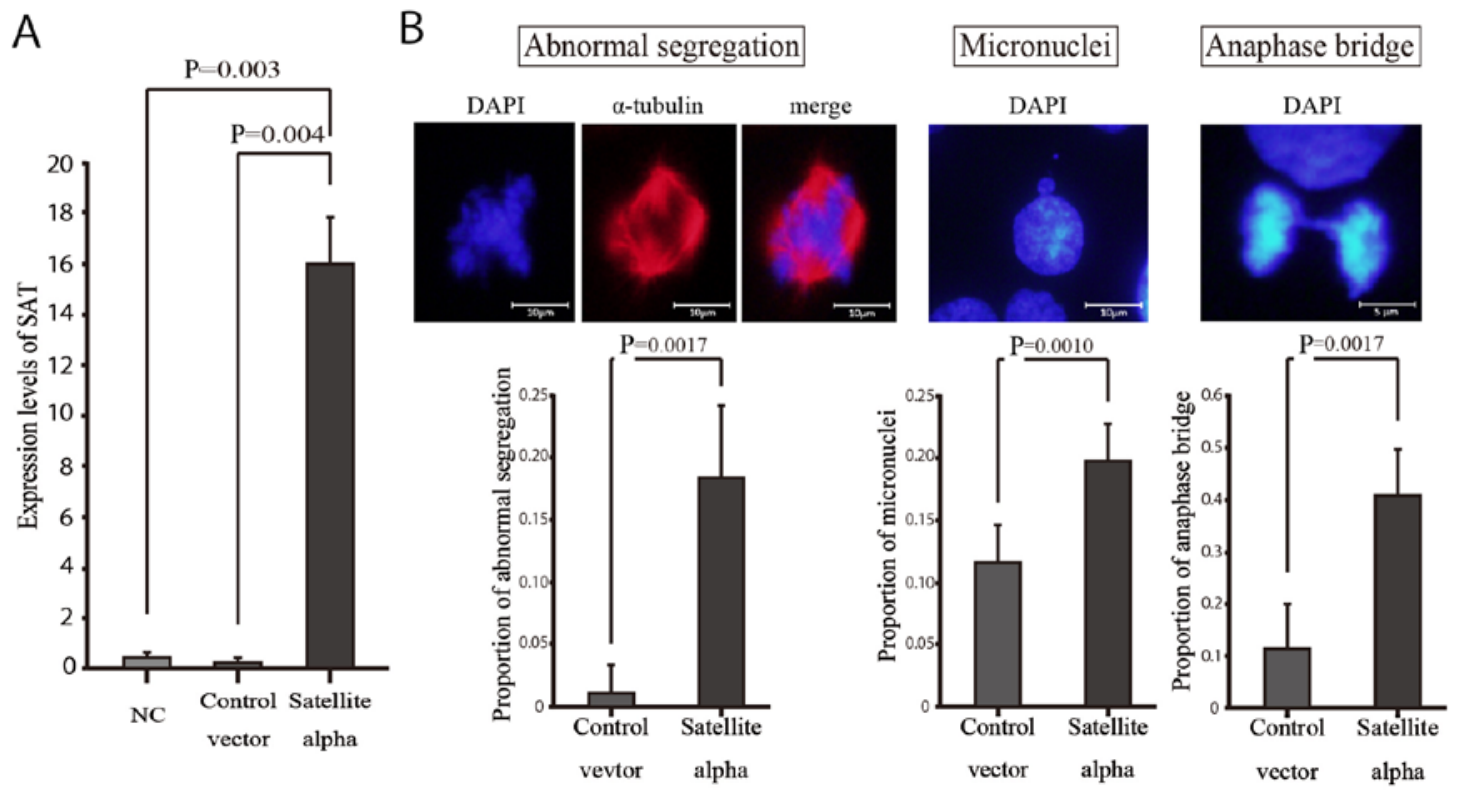

\section{Control vector}

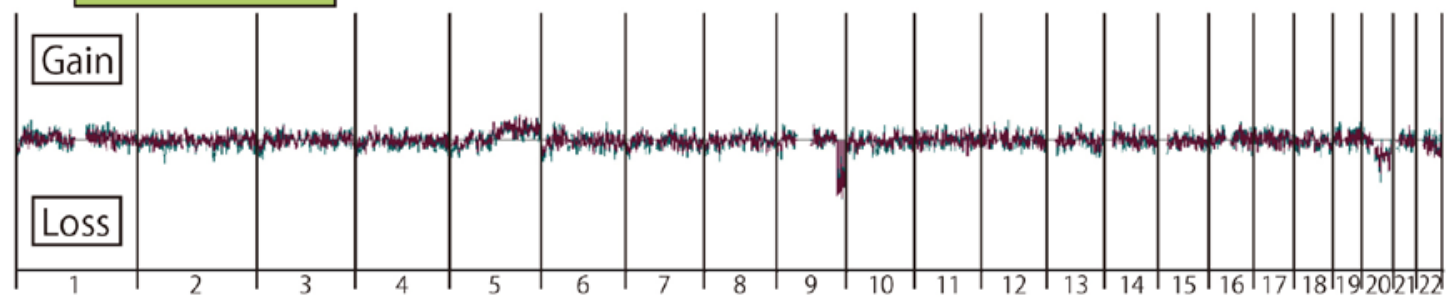

Satellite alpha

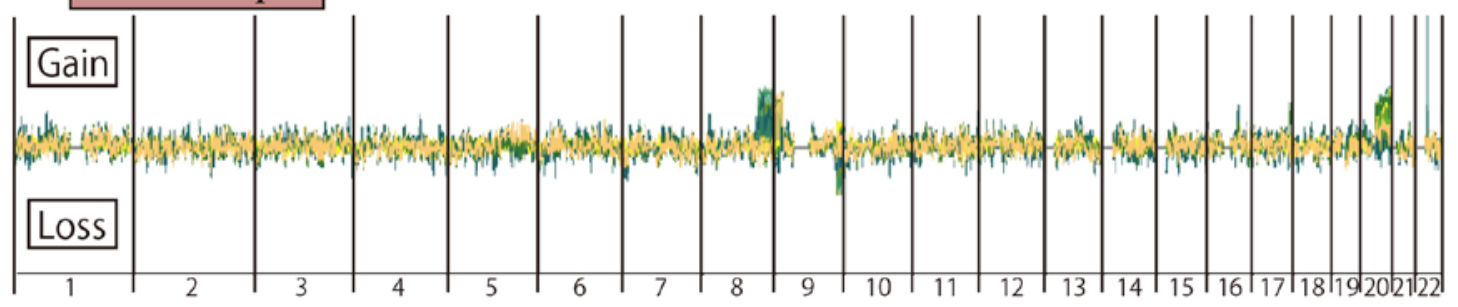

Figure 4. The overexpression of satellite alpha transcript (SAT) induces chromosomal instability in vitro. (A) A comparison of the expression of SAT between cells infected by satellite alpha-positive vectors and controls. NC, negative controls without infection. (B) Comparisons of the incidence of abnormal segregation, micronuclei and anaphase bridge between SAT-overexpressing cells and controls. The photographs represent typical images of each abnormal event. The incidence of abnormal segregation, micronuclei and anaphase bridge were investigated in 20 mitotic cells, 100 cells and 20 mitotic cells per sample, respectively. (C) Copy number alterations of autosomal chromosome detected by array CGH. Gains are shown in the upper area and losses in the lower area. Experimental samples are distinguished by different colors. Vertical lines indicate the boundaries of chromosomes. Numbers represent the chromosome number. In each chromosome area, the short arm is located on the left side, and the long arm is on the right side.

instability in normal mammary cells, we transduced the satellite alpha sequences using lentiviral vectors into HMEpCs and consequently induced the overexpression of SAT in these cells. According to the GFP-positive rates on flow cytometry (data not shown), the transduction efficiencies were 69.2 and $70.1 \%$ in the satellite alpha-transfected cells and control cells (transfected by vectors without satellite alpha sequences), respectively. RT-qPCR revealed a significantly higher expression of SAT in the cells transfected with satellite alpha-positive viruses than in the Control vector group (Fig. 4A).

Immunocytochemistry was performed to detect mitotic errors that might lead to chromosomal instability. On comparing transduced cells with satellite alpha sequences and those without such sequences, the rates of abnormal segregations of chromosomes, micronuclei and anaphase bridging were significantly higher in the cells with satellite alpha harboring overexpression of SAT than in those without it (Fig. 4B).

Array CGH in vitro with high and low SAT expression. To identify the specific locations at which the overexpression of SAT led to chromosomal instability, copy number alterations in satellite alpha-transfected cells were determined by array CGH (Fig. 4C). Copy number gains were frequently observed at specific locations in the long arm (q arm) of human chromosomes 8 and 20 ( $8 q$ and $20 q$ ) in the SAT-overexpressing cells, but not in the control vector-transfected cells, suggesting that these chromosomal alterations were induced by the overexpression of SAT. By contrast, copy number losses of chromosome $9 \mathrm{q}$ were observed in both cell groups, suggesting 
Table IV. Clinicopathological characeristics of breast cancer specimens in array CGH analyses.

\begin{tabular}{llllllllll}
\hline $\begin{array}{l}\text { Case } \\
\text { no. }^{\text {a }}\end{array}$ & $\begin{array}{c}\text { SAT } \\
\text { expression }\end{array}$ & Age & BMI & T & N & M & ER & HER2 & $\begin{array}{c}\text { Bilateral } \\
\text { breast tumors }\end{array} \begin{array}{c}\text { Malignancies } \\
\text { in other organs }\end{array}$ \\
\hline 1 & Low & 48 & 26 & 2 & - & - & - & - & - \\
2 & Low & 41 & 19 & 1 & - & - & + & - & - \\
3 & Low & 39 & 25 & 2 & + & - & - & - & - \\
4 & Low & 68 & 26 & 1 & - & - & + & + & - \\
41 & High & 34 & 19 & 1 & - & - & + & - & - \\
43 & High & 43 & 18 & 1 & - & - & + & + & - \\
44 & High & 59 & 19 & 2 & - & - & - & - & - \\
45 & High & 48 & 23 & 1 & + & - & + & + & - \\
\hline
\end{tabular}

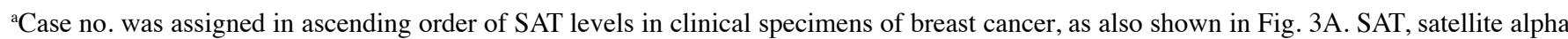
transcript; BMI, body mass index; ER, estrogen receptor; HER2, human epidermal growth factor receptor type 2.

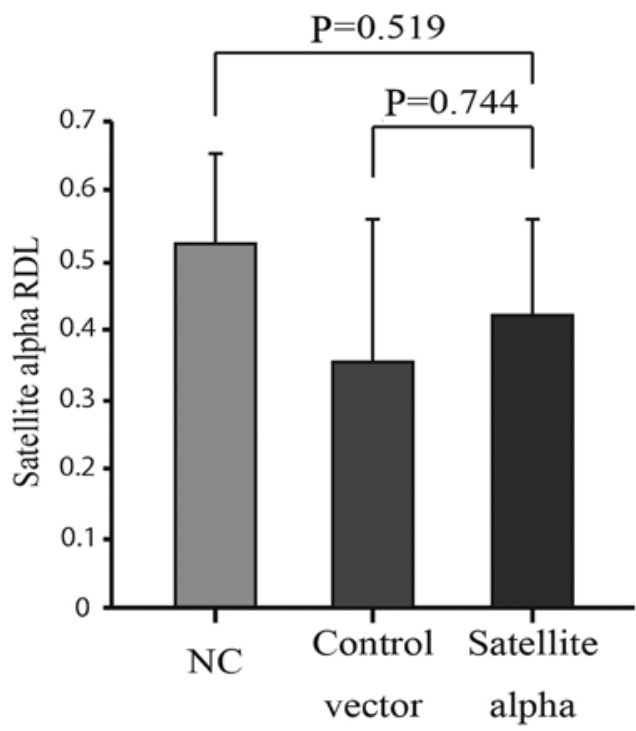

Figure 5. Comparisons of the RDL between SAT-overexpressing cells and controls. Error bars represent the standard deviation. RDL, relative demethylation level.

that those were differences from the reference DNA employed in the CGH arrays.

Alterations in the methylation levels in satellite alpha-transfected cells. Based on signal transduction, hypomethylation at the centromere leads to the overexpression of SAT; however, the reverse effect has not been examined. We therefore focused on the methylation levels at the centromere in connection with the expression of SAT. Using HMEpCs transduced with the satellite alpha sequences, the methylation levels of HMEpCs were measured via the MethyLight method and compared with those of control cells. No significant reduction in the methylation was observed in HMEpCs overexpressing SAT compared to both the Control vector and NC groups (Fig. 5). This indicates that increased levels of SAT did not affect the levels of methylation at the centromere, although decreased levels of methylation at the centromeric regions enhanced the expression of SAT.

\section{Discussion}

Chromosomal instability is induced by the overexpression of SAT, even in normal epithelial cells. The aberrant overexpression of satellite RNAs has been observed in various epithelial cancer cells (20) and may be actively involved in cellular carcinogenesis $(21,22)$. It also leads to chromosomal instability in BRCA1-knockout mammary gland cells and KRAS-mutated pancreatic epithelial cells $(21,22)$. However, its effects in normal cells have not yet been verified, at least to the best of our knowledge. In this study, we demonstrated that the overexpression of SAT led to chromosomal mis-segregations in normal mammary epithelial cells, enhancing chromosomal instability (Fig. 4B). To the best of our knowledge, this is the first report to demonstrate that chromosomal instability is induced by excessive satellite RNA in normal human epithelial cells.

Cells with chromosomal mis-segregations are considered to be at an increased risk of undergoing carcinogenesis, although they did not acquire malignant potential in the present study. These cells displayed contact inhibition following several rounds of subculture, as shown in the untransfected HMEpCs (data not shown). This implies that mis-segregation does not cause cells to transform, but instead encourages cell death by apoptotic machinery. Immunocytochemistry revealed representative morphological changes of mis-segregation during cell division (Fig. 4B), and array CGH revealed copy number alterations of specific chromosomes (Fig. 4C), both of which are strongly implicated in chromosomal instability. Such abnormal segregations observed in immunocytochemistry seemed to occur in wide and unspecified regions of chromosomes rather than in specific chromosomes. The overexpression of SAT may enhance the risk of abnormal segregation of cells in any region of the chromosome, but most cells are excluded by apoptotic machinery, as they lack the growth advantages characteristic of cancer cells. Surviving cells may escape this apoptotic machinery via SAT-induced copy number gains in chromosomes $8 \mathrm{q}$ and $20 \mathrm{q}$.

DNA hypomethylation and carcinogenesis. Decreased levels of methylation at the centromeric regions reportedly cause the 
overexpression of satellite RNA, which induces chromosomal instability (24-26). In this study, we demonstrated a significant correlation between hypomethylation at the satellite regions and the expression of SAT in clinical specimens of breast cancer (Fig. 2). By contrast, the overexpression of SAT did not affect the levels of methylation at the satellite regions (Fig. 5), indicating that a decrease in the levels of methylation occurs in advance at the centromeric regions, followed by the overexpression of SAT in the process of chromosomal instability. Therefore, hypomethylation plays an initial role in carcinogenesis. Nevertheless, epigenetic defects by themselves may not be sufficient to drive carcinogenesis, while the appropriate regulation of cell cycle checkpoints is in working order. For example, ICF syndrome caused by aberrant DNA methyltransferase $3 b$ (DNMT3b) leads to genome-wide hypomethylation, but it rarely induces malignant disease $(28,29)$. Our findings indicated that hypomethylation and SAT overexpression induced chromosomal mis-segregation in non-cancer cells, although these normal cells did not exhibit infinite proliferative capacity, suggesting that further contributions are required for the development of cancer.

Specific chromosome alterations in array CGH of clinical specimens. No significant differences in the number or size of the altered regions on a whole-genomic scale were observed between clinical samples with high and low expression of SAT in the array $\mathrm{CGH}$ analyses (Fig. 3C). We therefore focused on specific chromosomes, identifying obvious differences between them. Copy number gains at $8 \mathrm{q}$ and $20 \mathrm{q}$ were frequently observed only in clinical samples with a high expression of SAT, with no such observations made in those with a low expression, which was consistent with data obtained in vitro using satellite alpha-transfected cells (Figs. 3B and 4C). Some copy number alterations were also found in other chromosomal regions in samples with both high and low expression of SAT. Chromosomal alteration of $8 \mathrm{q}$ and $20 \mathrm{q}$ may have occurred at the initiation of breast cancer development prior to the progression of cancer, where these alterations may occur.

These chromosomal alterations are universally recognized in breast cancer (30). Of note, chromosomes $8 \mathrm{q}$ and $20 \mathrm{q}$ are so-called 'fragile regions' where aberrations are frequently found in several other carcinomas (31-33). Copy number gains in those specific chromosomes are observed from the very beginning of carcinogenesis in most types of carcinomas and are not specific to breast cancer. However, whether or not the copy number alterations at chromosomes $8 \mathrm{q}$ and $20 \mathrm{q}$ are essential for subsequent carcinogenesis is unclear. As shown in this study, the overexpression of SAT led to chromosomal alterations at chromosomes $8 \mathrm{q}$ and $20 \mathrm{q}$ in vitro, although no malignancy developed. Given that these chromosomal alterations are frequently observed in clinical specimens with a high expression of SAT, however, they may be involved in the development of cancer together with subsequent oncogenic genetic and epigenetic alterations in the process of acquiring malignant potential.

In conclusion, the overexpression of SAT impaired the stability of chromosomes $8 \mathrm{q}$ and $20 \mathrm{q}$ in normal mammary epithelial cells, as well as in clinical specimens of breast cancer. Chromosomal instability induced by the overexpression of SAT may be involved in the development of breast cancer. It is important, however, to interpret our results within the context of the study limitations, and further studies are required to draw definitive conclusions.

\section{Acknowledgements}

Not applicable.

\section{Funding}

This study was supported in part by a Grant-in-Aid for postgraduate students from Jichi Medical University and The JMU Graduate Student Start-Up Award, a Grant-in-Aid from the Ministry of Education, Culture, Sports, Science and Technology, and the JKA Foundation through its promotion funds from Keirin Racing.

\section{Availability of data and materials}

The analyzed datasets generated during the study are available from the corresponding author on reasonable request.

\section{Authors' contributions}

KI designed the study and wrote the initial draft of the manuscript. KS contributed to analysis and interpretation of data and assisted in the preparation of the manuscript. TO contributed to the execution of the lentiviral infection experiments. TF, YT, NK, FW, HI, YM, TK, MS, KF, YM, HN, FK and TR contributed to data collection and interpretation, and critically reviewed the manuscript. All authors have read and approved the final manuscript.

\section{Ethics approval and consent to participate}

This study was approved by the Research Ethics Committee at Jichi Medical University. Written informed consent was obtained from each study participant.

\section{Consent for publication}

Not applicable.

\section{Competing interests}

The authors declare that they have no competing interests.

\section{References}

1. Feinberg AP and Vogelstein B: Hypomethylation distinguishes genes of some human cancers from their normal counterparts. Nature 301: 89-92, 1983.

2. Rodriguez J, Frigola J, Vendrell E, Risques RA, Fraga MF, Morales C, Moreno V, Esteller M, Capellà G, Ribas M, et al: Chromosomal instability correlates with genome-wide DNA demethylation in human primary colorectal cancers. Cancer Res 66: 8462-9468, 2006.

3. Herrera LA, Prada D, Andonegui MA and Dueñas-González A: The epigenetic origin of aneuploidy. Curr Genomics 9: 43-50, 2008.

4. Kawano H, Saeki H, Kitao H, Tsuda Y, Otsu H, Ando K, Ito S, Egashira A, Oki E, Morita M, et al: Chromosomal instability associated with global DNA hypomethylation is associated with the initiation and progression of esophageal squamous cell carcinoma. Ann Surg Oncol 21 (Suppl 4): S696-S702, 2014. 
5. Esteller M, Silva JM, Dominguez G, Bonilla F, Matias-Guiu X, Lerma E, Bussaglia E, Prat J, Harkes IC, Repasky EA, et al: Promoter hypermethylation and BRCA1 inactivation in sporadic breast and ovarian tumors. J Natl Cancer Inst 92: 564-569, 2000.

6. Yang B, Guo M, Herman JG and Clark DP: Aberrant promoter methylation profiles of tumor suppressor genes in hepatocellular carcinoma. Am J Pathol 163: 1101-1107, 2003.

7. Herman JG and Baylin SB: Gene silencing in cancer in association with promoter hypermethylation. N Engl J Med 349: 2042-2054, 2003.

8. Itano O, Ueda M, Kikuchi K, Hashimoto O, Hayatsu S, Kawaguchi M, Seki H, Aiura K and Kitajima M: Correlation of postoperative recurrence in hepatocellular carcinoma with demethylation of repetitive sequences. Oncogene 21: 789-797, 2002.

9. Widschwendter M, Jiang G, Woods C, Müller HM, Fiegl H, Goebel G, Marth C, Müller-Holzner E, Zeimet AG, Laird PW, et al: DNA hypomethylation and ovarian cancer biology. Cancer Res 64: 4472-4480, 2004.

10. Igarashi S, Suzuki H, Niinuma T, Shimizu H, Nojima M, Iwaki H, Nobuoka T, Nishida T, Miyazaki Y, Takamaru H, et al: A novel correlation between LINE-1 hypomethylation and the malignancy of gastrointestinal stromal tumors. Clin Cancer Res 16: 5114-5123, 2010.

11. Eden A, Gaudet F, Waghmare A and Jaenisch R: Chromosomal instability and tumors promoted by DNA hypomethylation. Science 300: 455, 2003

12. Gaudet F, Hodgson JG, Eden A, Jackson-Grusby L, Dausman J, Gray JW, Leonhardt H and Jaenisch R: Induction of tumors in mice by genomic hypomethylation. Science 300: 489-492, 2003.

13. Suzuki K, Suzuki I, Leodolter A, Alonso S, Horiuchi S, Yamashita K and Perucho M: Global DNA demethylation in gastrointestinal cancer is age dependent and precedes genomic damage. Cancer Cell 9: 199-207, 2006.

14. Saito M, Suzuki K, Maeda T, Kato T, Kamiyama H, Koizumi K, Miyaki Y, Okada S, Kiyozaki H and Konishi F: The accumulation of DNA demethylation in Sat $\alpha$ in normal gastric tissues with Helicobacter pylori infection renders susceptibility to gastric cancer in some individuals. Oncol Rep 27: 1717-1725, 2012.

15. Plohl M, Meštrović N and Mravinac B: Centromere identity from the DNA point of view. Chromosoma 123: 313-325, 2014

16. Ugarkovic D: Functional elements residing within satellite DNAs. EMBO Rep 6: 1035-1039, 2005.

17. Ideue T, Cho Y, Nishimura $\mathrm{K}$ and Tani T: Involvement of satellite I noncoding RNA in regulation of chromosome segregation. Genes Cells 19: 528-538, 2014

18. Rošić S, Köhler F and Erhardt S: Repetitive centromeric satellite RNA is essential for kinetochore formation and cell division. J Cell Biol 207: 335-349, 2014.

19. McNulty SM, Sullivan LL and Sullivan BA: Human centromeres produce chromosome-specific and array-specific alpha satellite transcripts that are complexed with CENP-A and CENP-C. Dev Cell 42: 226-240.e6, 2017.

20. Ting DT, Lipson D, Paul S, Brannigan BW, Akhavanfard S, Coffman EJ, Contino G, Deshpande V, Iafrate AJ, Letovsky S, et al: Aberrant overexpression of satellite repeats in pancreatic and other epithelial cancers. Science 331: 593-596, 2011.

21. Zhu Q, Pao GM, Huynh AM, Suh H, Tonnu N, Nederlof PM, Gage FH and Verma IM: BRCA1 tumour suppression occurs via heterochromatin-mediated silencing. Nature 477: 179-184, 2011.
22. Kishikawa T, Otsuka M, Yoshikawa T, Ohno M, Ijichi H and Koike K: Satellite RNAs promote pancreatic oncogenic processes via the dysfunction of YBX1. Nat Commun 7: 13006, 2016.

23. Lehnertz B,Ueda Y, Derijck AA, Braunschweig U, Perez-Burgos L, Kubicek S, Chen T, Li E, Jenuwein T and Peters AH: Suv39hmediated histone $\mathrm{H} 3$ lysine 9 methylation directs DNA methylation to major satellite repeats at pericentric heterochromatin. Curr Biol 13: 1192-1200, 2003.

24. Bouzinba-Segard H, Guais A and Francastel C: Accumulation of small murine minor satellite transcripts leads to impaired centromeric architecture and function. Proc Natl Acad Sci USA 103: 8709-8714, 2006.

25. Eymery A, Horard B, El Atifi-Borel M, Fourel G, Berger F, Vitte AL, Van den Broeck A, Brambilla E, Fournier A, Callanan M, et al: A transcriptomic analysis of human centromeric and pericentric sequences in normal and tumor cells. Nucleic Acids Res 37: 6340-6354, 2009.

26. Leonova KI, Brodsky L, Lipchick B, Pal M, Novototskaya L, Chenchik AA, Sen GC, Komarova EA and Gudkov AV: p53 cooperates with DNA methylation and a suicidal interferon response to maintain epigenetic silencing of repeats and noncoding RNAs. Proc Natl Acad Sci USA 110: E89-E98, 2013.

27. Weisenberger DJ, Campan M, Long TI, Kim M, Woods C, Fiala E, Ehrlich M and Laird PW: Analysis of repetitive element DNA methylation by MethyLight. Nucleic Acids Res 33: 6823-6836, 2005.

28. Hagleitner MM, Lankester A, Maraschio P, Hultén M, Fryns JP, Schuetz C, Gimelli G, Davies EG, Gennery A, Belohradsky BH, et al: Clinical spectrum of immunodeficiency, centromeric instability and facial dysmorphism (ICF syndrome). J Med Genet 45: 93-99, 2008.

29. Walton EL, Francastel C and Velasco G: Dnmt3b prefers germ line genes and centromeric regions: Lessons from the ICF Syndrome and Cancer and Implications for Diseases. Biology (Basel) 3: 578-605, 2014.

30. Tsuji K, Kawauchi S, Saito S, Furuya T, Ikemoto K, Nakao M, Yamamoto S, Oka M, Hirano T and Sasaki K: Breast cancer cell lines carry cell line-specific genomic alterations that are distinct from aberrations in breast cancer tissues: Comparison of the CGH profiles between cancer cell lines and primary cancer tissues. BMC Cancer 10: 15, 2010

31. Uchida M, Tsukamoto Y, Uchida T, Ishikawa Y, Nagai T, Hijiya N, Nguyen LT, Nakada C, Kuroda A, Okimoto T, et al: Genomic profiling of gastric carcinoma in situ and adenomas by array-based comparative genomic hybridization. J Pathol 221: 96-105, 2010.

32. Tabach Y, Kogan-Sakin I, Buganim Y, Solomon H, Goldfinger N, Hovland R, Ke XS, Oyan AM, Kalland KH, Rotter V, et al: Amplification of the $20 \mathrm{q}$ chromosomal arm occurs early in tumorigenic transformation and may initiate cancer. PLoS One 6: e14632, 2011

33. Orsetti B, Selves J, Bascoul-Mollevi C, Lasorsa L, Gordien K, Bibeau F, Massemin B, Paraf F, Soubeyran I, Hostein I, et al: Impact of chromosomal instability on colorectal cancer progression and outcome. BMC Cancer 14: 121, 2014. 